\title{
Distributed sensing and cooperative estimation/detection of ubiquitous power internet of things
}

\author{
Haoyong Chen ${ }^{1 *}$ D, Xiaojuan Wang ${ }^{1}$, Zhihao Li $^{1}$, Wutao Chen ${ }^{1}$ and Yongzhi Cai ${ }^{2}$
}

\begin{abstract}
The rapid development of Internet Plus Smart Energy requires further strengthening of three kinds of interconnections based on traditional power systems: physical interconnection, information interconnection, and commercial interconnection. Due to the integration of renewable energy, the reform of the electricity market, and the deployment of the Smart Grid, a large amount of data will be generated. Thus, it is necessary to establish a Ubiquitous Power Internet of Things (UPIOT) to realize connections among people and things, things and things, and people and people in power systems. This paper studies the concept and architecture of the UPIoT and indicates the deployment of the perception layer and network layer as the key to building UPIoT in the initial stage. As UPloT tends to cover a wide area and produce massive and distributed data, signal processing and data analytics theories and techniques are needed to handle the data and observe the state of the large-scale system. Further studies on distributed sensing and cooperative estimation theories and techniques of UPIoT are also required. Finally, the application prospects of UPIoT and the directions for future research are discussed.
\end{abstract}

Keywords: Ubiquitous power internet of things, Ubiquitous perception, Distributed sensing, Cooperative estimation, Cooperative detection

\section{Introduction}

The concept of Energy Internet (which is also called Internet Plus Smart Energy in China) was proposed by the famous American futurist Jeremy Rifkin. The basic characteristics of Internet Plus Smart Energy include using renewable energy as the main primary energy source, allowing the access of ultra-large-scale distributed generation systems and distributed energy storage systems, realizing wide-area energy sharing based on Internet technology, and supporting the electrification of transportation systems.

With the further development of Internet Plus Smart Energy, the future trend of power (energy) grids is to strengthen the three interconnections, namely, physical interconnection, information interconnection, and commercial interconnection. Based on the existing power grid, the first step is to strengthen the physical interconnection

\footnotetext{
* Correspondence: eehychen@scut.edu.cn

${ }^{1}$ School of Electric Power, South China University of Technology, Guangzhou, China

Full list of author information is available at the end of the article
}

to achieve the integration and comprehensive utilization of multiple forms of energy (e.g., electricity, heat, cold, gas, and so on), to form integrated energy systems. The second step is to strengthen the information interconnection, which is established in part by the Internet of Things (IoT), to achieve smooth communication anytime/anywhere and among anybody/anything based on more advanced information and communication technology. The final step is then to strengthen the commercial interconnection to connect the supply and demand sides through market mechanisms and higher level sharing economic models.

With the advancement of energy production and the consumption revolution, traditional energy service providers are gradually transforming into customer-centered integrated energy service providers. At present, one hot trend in energy development is to build an integrated energy system with multi-energy forms to optimize their complementarity and respond to the national "Internet Plus Smart Energy" initiative. On March 8, 2018, the State Grid Corporation of China made comprehensive arrangements 
for the construction of Ubiquitous Power Internet of Things (UPIoT) and has since accelerated the implementation of the strategy of "Three Types \& Two Networks" (hub type, platform type, shared type, strong Smart Grid, and UPIoT). UPIoT realizes the interconnection of things and people in every link of power systems. Strong Smart Grid and UPIoT complement each other and can be developed together to form a powerful value creation platform, constituting a system of Internet Plus Smart Energy that combines energy flow, business flow, and data flow. In 2015, the No. 9 document of the Central Committee of the Communist Party of China launched the new round of electricity market reform, which aims to turn the power grid into an electric power energy channel and achieve independent choice in both the supply and demand sides. However, there exists a risk of being "pipelined" for the power grid. Due to the integration of renewable energy, the reform of the electricity market, and the development of the Smart Grid and Internet Plus Smart Energy, a large amount of data will be generated. Although these data would have significant value, it is currently not being collected and used. The original intention of the State Grid Corporation of China in developing UPIoT was to utilize these data using the company's power grid infrastructure and unique data resources to cultivate advanced services and form core competitiveness at a higher level.

Communication among people and things, things and things, and people and people is considered to be one of the prominent features of ubiquitous networks. The rapid development of wireless, broadband, and Internet technologies is continuously deepening ubiquitous network applications [1], as shown in Fig. 1. In recent years, there have been many studies on IoT. A safe and efficient data collection scheme is proposed in $[2,3]$ outlines the related technologies of IoT, focusing on its technologies, protocols, and application scenarios. Different application scenarios of IoT in the Smart Grid are described in [4], whereas [5] explores the application of big data in IoT and outlines key issues to be resolved for future research. Reference [6] focuses on the definition of Power Internet of Things and the main technical features of the perception layer, network layer, and application layer in its network architecture. The connotation, key technologies, definitions, and modeling methods of the sensor information model of the sensory layer are elaborated in [7]. However, the introduction and the related key technologies of UPIoT have not been well defined.

This paper studies the current situation of UPIoT and indicates that the perception layer and network layer, as the key elements to building UPIoT, need to be completed prior to the next stage of construction. This study also explores the basic theories of distributed sensing and collaborative estimation/detection [8] and describes the application prospects of UPIoT.

\section{Ubiquitous power loTs}

The goal of UPIoT is to fully apply modern information technologies and advanced communication technologies, such as mobile internet and artificial intelligence, to all aspects of power systems in order to realize the

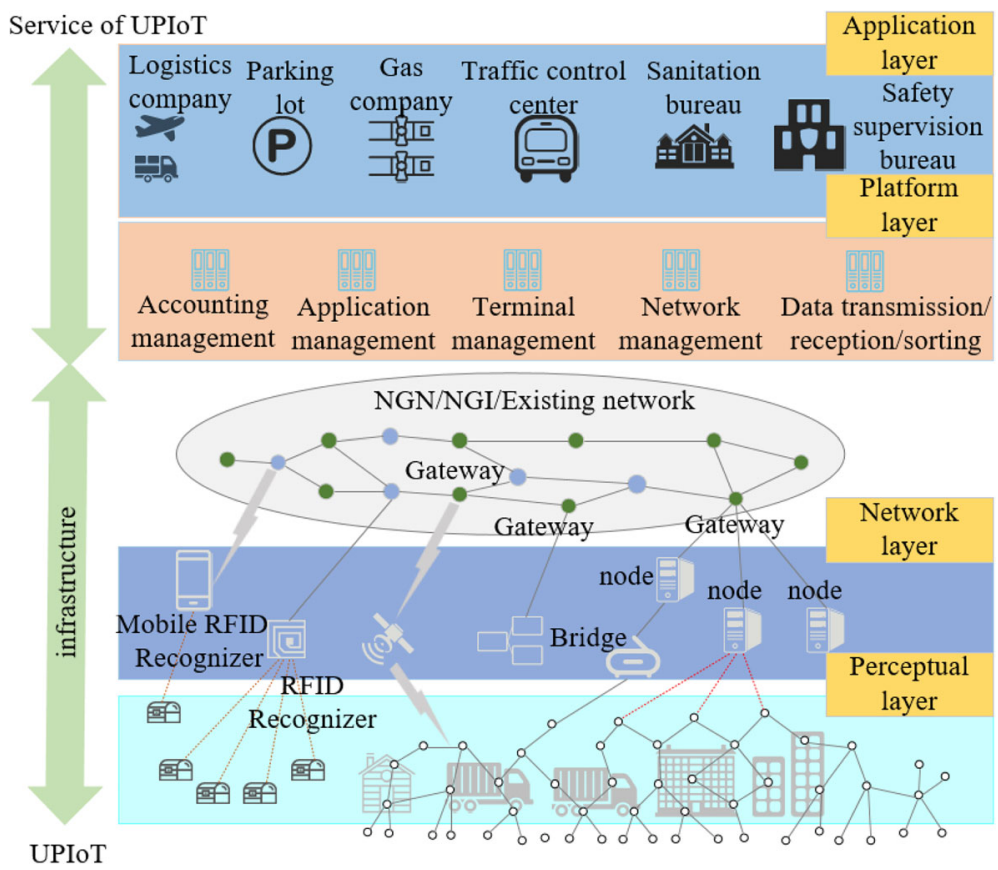

Fig. 1 Ubiquitous network diagram 
interconnection of all things and human-computer interaction within the systems. UPIoT is an important part of Internet Plus Smart Energy, and it acts as a smart service system with comprehensive state awareness, efficient information processing, and convenient and flexible application features. The transition from the concept of Smart Grid to the specific scheme of UPIoT is a natural evolution of establishing the basis of information interconnection. "Ubiquitous" means existing everywhere, and although the existing power grid is a ubiquitous network, its ubiquity is only embodied in the electric energy. The ubiquity of power grids cannot be reflected in terms of data or information, whereas UPIoT aims to achieve the ubiquity of data and information by sinking the basic data connection. The basic categories of data include user electrical data, power system's edge data, and smart energy new business data. Although the data in each of these categories are considered to be small data compared with the traditional measurement data, the intelligent connections of a massive number of small data sets can achieve ubiquitous perception. Figure 2 displays a framework diagram of the Internet Plus Smart Energy, showing that the
Smart Grid is a combination of information networks and physical networks. The connection and data in each production link become one of the key driving factors of the Internet Plus Smart Energy.

UPIoT is based on the general IoT, and Fig. 3 shows the relationship among the ubiquitous network, the Internet of Things, and the sensor network [9]. Due to the integration of renewable energy, the reform of the electricity market, and the deployment of the Smart Grid or Internet Plus Smart Energy, a large amount of data are generated. As previously described, although the data are basically "small data" and the value of a single datum is low, the collection of massive data has great value, and UPIoT is an important way to achieve the connections. The collection of massive data mainly includes small data connections on the power user side and in all aspects of power transmission and distribution. There are many kinds of small data on the user side, generated by energy consumers such as households, various buildings, agricultural, industrial, and commercial enterprises, and public infrastructure. The small data that are transmitted and distributed mainly include environmental data, device status data, and affiliated facility status data.

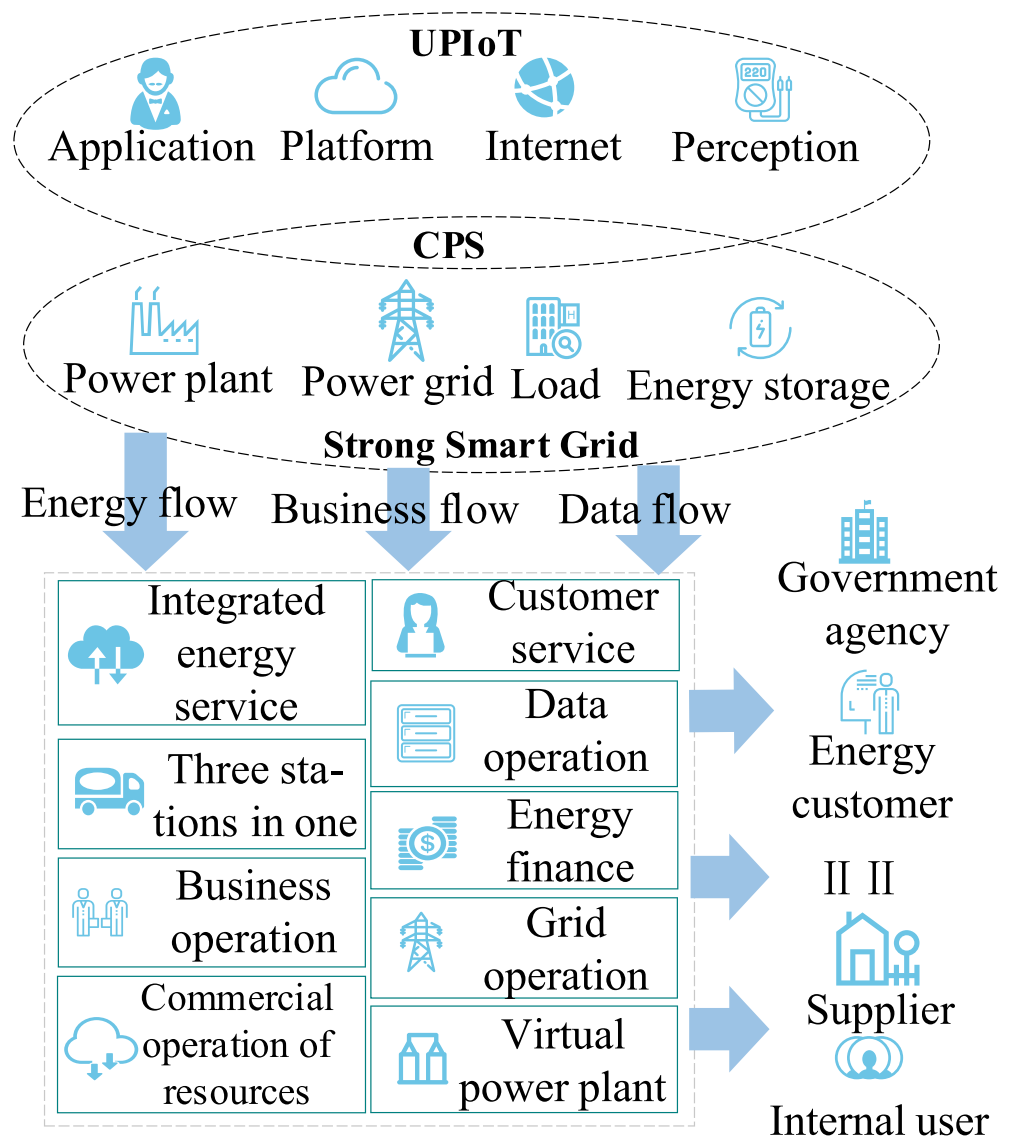

Fig. 2 Framework of the Internet Plus Smart Energy 


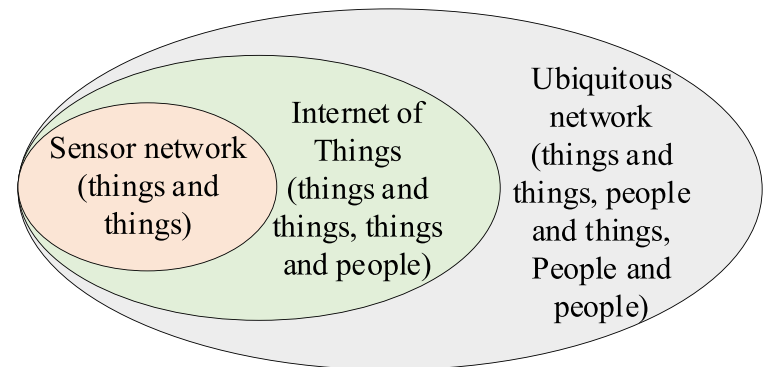

Fig. 3 The relationship among the ubiquitous network, loT, and the sensor network

UPIoT includes the perception layer, network layer, platform layer, and application layer [10], as shown in Fig. 4.

1) Perception layer. The perception layer is the lowest layer of the conventional architecture of UPIoT, and its main responsibility is to collect useful information/data from things or environment and transform them in a digital setup. The perception layer mainly consists of various sensors and smart meters deployed in electrical equipment, transmission lines, and affiliated facilities.

2) Network layer. The network layer mainly realizes the transmission of data collected by various types of sensors in power systems at the perception layer to the application layer (service processing end), including public communication network, private power wired/wireless communication networks, and so on.

3) Platform layer. The platform layer is a public infrastructure platform, which realizes the management of various types of UPIoT data, the UPIoT network, the IoT operating systems, and so on. The value of massive data is deeply explored, and Application Programming Interface (API) for application development will also be provided.

4) Application layer. The application layer is the highest layer of UPIoT, which uses cloud computing technology, virtualization technology, data mining technology, and so on. to realize centralized storage and deep mining of data collected by the perception layer and provide intelligent services or visual presentation.

The perception layer of UPIoT mainly collects, identifies, and aggregates data by various sensory identification devices [11]. IoT architecture includes data transmission from the front-end intelligent perception layer terminals to IoT communication modules and then from the communication modules to the base station, which is the core technology of IoT wireless communication. After collecting data, the base station can upload it to the cloud server platform through the traditional 3G, 4G, and $5 \mathrm{G}$ networks or private power wired/wireless communication networks. Based on the cloud platform, a variety of business applications can then be established. The above is the complete route from the perception layer to the network layer, the platform layer, and finally the application layer. As IoT develops gradually and UPIoT is still in the preliminary deployment stage, the current development of UPIoT should focus on the perception layer and the network layer. Only after the foundations of these two layers have been built can the platform layer and application layer be established.

\section{Distributed sensing and cooperative estimation/ detection of UPIoT}

3.1 Network architecture

Because UPIoT tends to cover a relatively wide area and produce massive and distributed data, signal processing

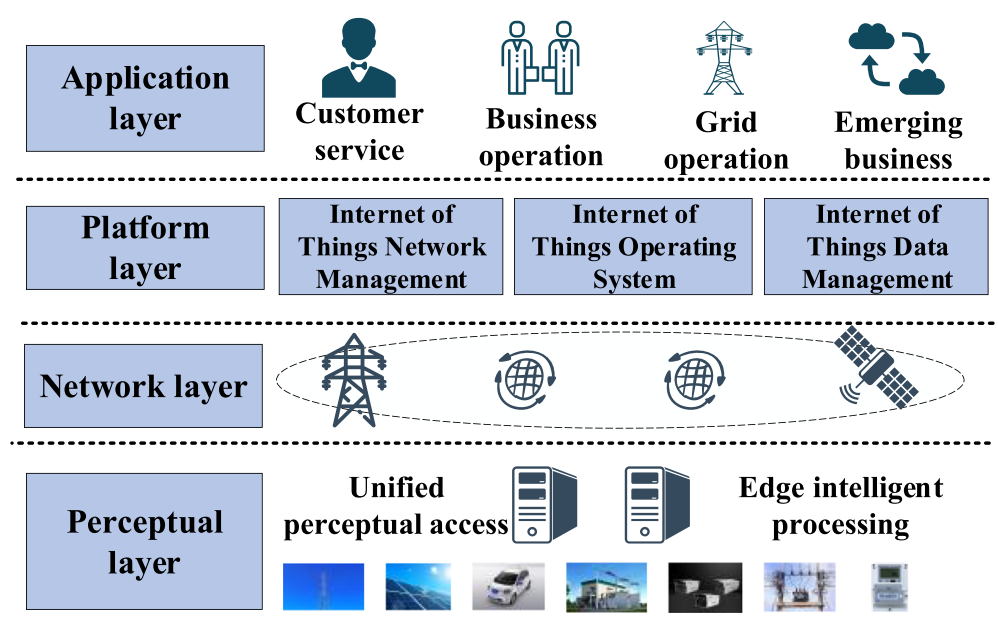

Fig. 4 Architecture of UPIOT 
and data analytics theories and techniques are needed to handle the data and observe the state of the large-scale system on which it resides. The distributed signal processing theories of UPIoT, which include distributed sensing and cooperative estimation/detection, are discussed here.

The signal processing architecture mainly includes centralized architecture, decentralized architecture, distributed architecture, and hybrid architecture [12, 13]. The optimal architecture is selected according to different objectives as follows:

1) Centralized architecture: It is the most common architecture in practical application, which is relatively simple in structure and relatively mature in algorithms and techniques but has high requirements for bandwidth and computing power.

2) Decentralized architecture: It is used when the system consists of different sub-systems. The sensor nodes in the sub-systems communicate with each other, and the fusion processing centers in each sub-system also communicate with others in different sub-systems. Thus, the communication burden across the sub-systems is reduced, but the internal communication burden within each sub-system is heavy.

3) Distributed architecture: There is no local fusion processing center, and each sensor node transmits the estimation results to the adjacent sensor node, which are also used to correct the estimation results. The advantages are that the communication cost is low, and the network scalability is good, and the edge computing may play an important role in this situation.

4) Hybrid architecture: The hybrid computing architecture with overall decentralized and locally distributed processing can also be employed.

\subsection{Theories of distributed sensing and cooperative estimation/detection \\ 3.2.1 Distributed sensing}

In distributed sensing [14], a group of sensors collectively observe information of the state of the environment. Due to factors such as cost, spectrum bandwidth limitation, and complexity, the sensors compress their own observation data and transmit only part of the information to the fusion center to estimate the state of the environment. Compared with centralized sensing, distributed sensing will lose some measurement information, and consequently, unprocessed sensing information can make the fusion center difficult to handle in large-scale systems, leading to real-time performance deterioration or even collapsing.

Distributed sensor systems have many different types of network structures. They usually form a connected graph with no loops, so information transmits along the uniquely determined path from the sensor node to the fusion center. Another type is the parallel structure in which each sensor node communicates directly with the fusion center. There is also a random configuration with sensor nodes randomly placed in the area cooperating with each other to form a dynamic communication network. The most common data transmission models of wireless sensor networks (WSNs) are shown in Fig. 5 [15].

The ideal distributed perception architecture is that each node in the network has equal status and can be used as a sensor, a router, a repeater, and so on [12]. A sensor located in the target event area senses its information and transmits the information to the fusion center through a single hop or multiple hops. Other nodes can be used as repeaters or routers, and the fusion center estimates the final results after one or more data transmissions.

\subsubsection{Cooperative estimation/detection}

The commonly used estimation methods for static estimation (Fig. 6) are the moment estimation method, maximum likelihood estimation, least squares method, Bayesian estimation, and so on. The most commonly used estimation method for dynamic estimation is the Kalman filtering method [16].

The distributed feature of UPIoT is that different sensors jointly collect state information in an area. Because of bandwidth and power limitations, the observed information needs to be quantized, and the collected signal is compressed. According to existing studies, the challenges of UPIoT-based estimation can be summarized as follows [12, 1) how to reduce the dimension to compress the
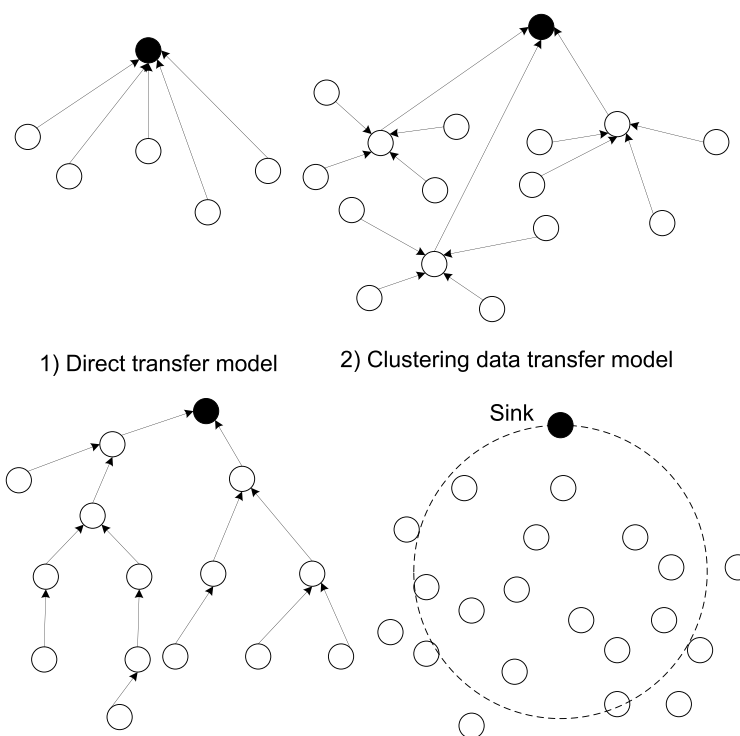

2) Clustering data transfer model

3) Planar Ad-hoc data transfer model

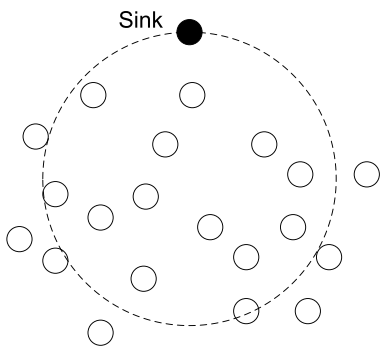

4) Mobile sink model

Fig. 5 Data transmission model of wireless sensor network 


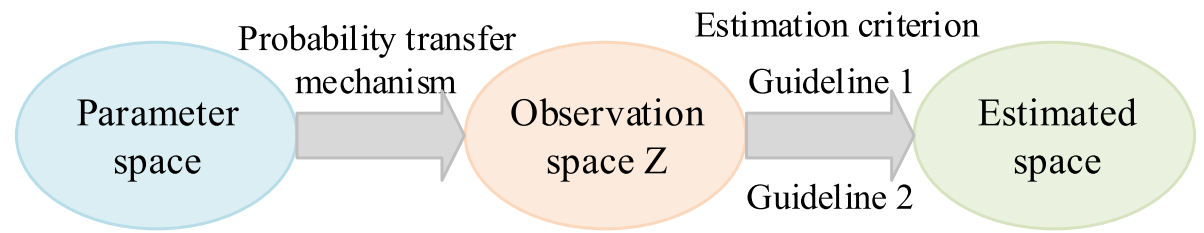

Fig. 6 Statistical model of parameter estimation

observation data, while maintaining the mean square error; (2) how to quantize the compressed signal before transmission; and (3) how to construct an estimator to quantize the compressed information. The theories for data compression and cooperative detection/estimation used in UPIoT are further introduced here.

\section{A. Data compression}

With the development of Internet Plus Smart Energy, the scale of the system and the requirements for communication systems are getting increasingly larger and higher. Thus, UPIoT may face the problem of insufficient computing power, storage capacity, transmission capacity, and power supply capacity. How to compress information and reduce the communication burden has become one of the key issues in current research $[17,18]$.

At present, the sparse technology commonly used in complex power systems reduces storage pressure by only storing non-zero elements, but as the system scale increases, sparse technology cannot meet the demand. In 2006, Candes et al. proposed a compressed sensing technology with which the signal is sparse or can be sparsely represented after transformation and can be sampled with a sampling frequency far lower than the Nyquist signal to obtain a compressed signal [19]. Therefore, perception and compression are completed together in one step. The comparison of the two processes is shown in Fig. 7.

\section{B. Cooperative detection and cooperative estimation}

In an $N$-node system, assuming a change at time $\lambda=k$, the observed values $X_{1}^{i}, \cdots, X_{k-1}^{i}$ on sensor $i$ are independent and identically distributed, whereas observation $X_{k}^{i}$,
$X_{k+1}^{i}$ follows another distribution. The observations of each sensor are independent of each other. When $t=n$, the observation information for sensor node $i$ is $X_{n}^{i}=\left[X_{1}^{i}, \cdots, X_{n}^{i}\right]$ , and the detection problem can be equivalent to the hypothesis test problem of " $\mathrm{H}_{0}: \lambda>n$ " and " $\mathrm{H}_{1}: \lambda \leq n$ ". If $\mathrm{H}_{0}$ is true, no change occurs, and if $\mathrm{H}_{0}$ is false, change occurs. In cooperative detection, in the absence of a fusion or control center, each sensor detects changes within the region in a parallel or distributed manner and interacts with information through a certain data transfer model. Finally, based on the information collected by itself and the interactive information, the local system can be detected. When there is a fusion or control center, the observations of all sensors are available at the fusion or control center. The cooperative detection framework is shown in Fig. 8.

After detecting changes in the system, it is necessary to estimate the observations to obtain the system status. Cooperative estimation means that in distributed networks the nodes exchange information with each other and update and correct their own information according to the exchanged information [20]. Cooperative estimation has the following advantages over centralized estimation [21-23]: cooperative estimation of each node's independent information and exchanging information with neighboring nodes can effectively reduce the computational time and increase the robustness of the network.

Considering a WSN system consisting of $N$ sensors, here we try to estimate a deterministic parameter $S$. Since the fusion center receives the quantitative observations, the goal is to obtain $\hat{S}$ with the least square error based on this quantitative observation. The $n$th sensor can observe that

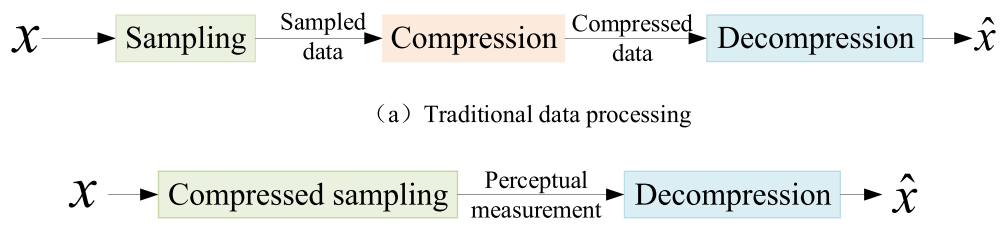

(b) Compressed sensing

Fig. 7 Comparison of data compression and compression perception 


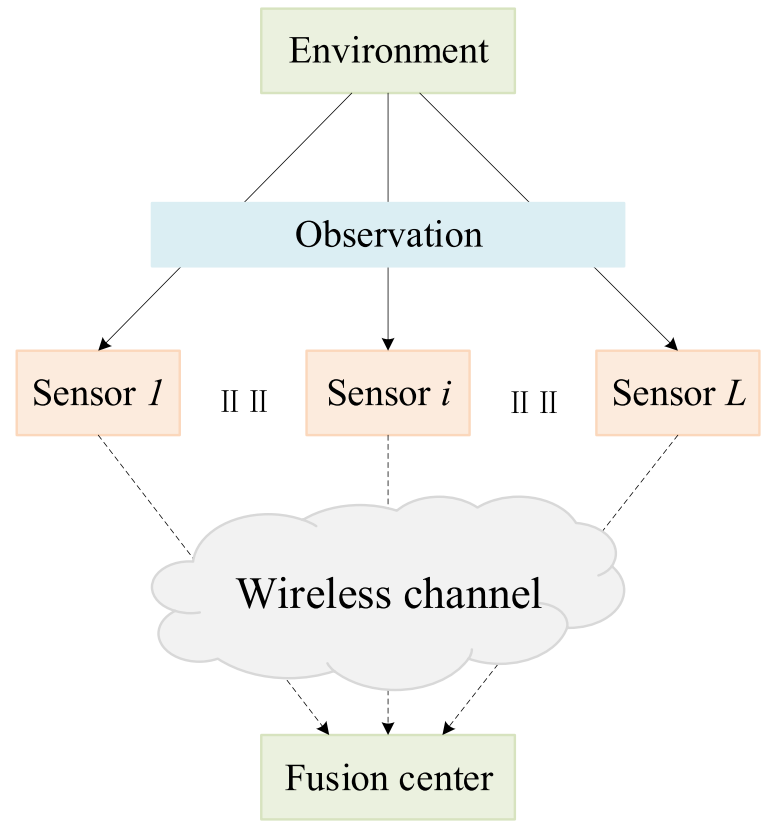

Fig. 8 Cooperative detection framework

$$
x(n)=s+w(n), n \in(0, N-1)
$$

where $w(n)$ is Gaussian white noise, which obeys a $\left(0, \sigma^{2}\right)$ distribution, and the $w(n)$ functions of different sensors are independent and identically distributed.

The quantified indicators are constructed as

$$
b_{k}(n)=1\left\{x(n) \in B_{k}(n)\right\}, k \in[1, K]
$$

which takes a value of one when $x(n) \in B_{k}(n) \subset R^{M}$ is satisfied; otherwise, it takes a value of zero.

After the signal is quantized, the network transmission path is used to transmit the quantized information to other nodes. After the information is exchanged, the node information is updated, and a suitable distributed algorithm is selected to cooperatively estimate the deterministic parameter $S$.

The ideal distributed estimation has the following characteristics [24]:

1) Accurate estimation. After partitioning the geographically distributed large-scale system, even when the system measurement value is not objective, each area can still accurately estimate the system operation status.

2) Small communication burden so real-time requirements can be met.

3) Distributed architecture with accuracy like that of the centralized estimation.

4) Good real-time performance due to the small amount of calculation required.

5) High robustness.

\section{Application prospects}

The main objective of UPIoT was to realize the sinking of the basic data connections. Such basic data mainly include small data connections on the electric power user side and in the electric power transmission and distribution networks:

1) There are many types of small data on the user side, such as data generated by smart meters, smart appliances, smart charging posts, and leakage monitoring, as well as data/control for energy efficiency management, intelligent buildings, and intelligent fire protection.

2) The small data of transmission and distribution networks include environmental data (temperature, humidity, and so on), device status data (temperature, dielectric strength, and so on), affiliated facility status data (cable tunnel environmental data, and so on), and other data.

After the data collection is completed, it is possible to establish corresponding services, such as optimizing contract energy management (energy saving service), based on the power demand data. UPIoT can also become a basic platform to build innovative value-added services, such as integrated energy services, Internet finance, big data operations, big data credit, photovoltaic cloud networks, online supply chain finance, virtual power plants, and new energy services, based on blockchain and smart manufacturing.

Because the deployment and application of UPIoT are interdisciplinary problems, the theories and techniques of signal processing, communication, data science, economics, and management science are needed.

\section{Conclusion}

The concept and architecture of Ubiquitous Power Internet of Things (UPIoT) were discussed in this study. UPIoT is the infrastructure for the development of Internet Plus Smart Energy, and it can be divided into the perception layer, network layer, platform layer, and application layer. Only after the establishment of the perception and network layers can UPIoT be further constructed. The theories of distributed sensing and cooperative estimation of UPIoT were introduced, which were needed to handle the data and observe the state of the large-scale system on which it resides. Finally, the application prospects of UPIoT and directions for future research were discussed.

\footnotetext{
Abbreviations

API: Application Programming Interface; loT: Internet of Things;

UPIOT: Ubiquitous Power Internet of Things
}

Acknowledgements

Not applicable. 


\section{Authors' contributions}

HYC as a corresponding author, contributed significantly to analysis, manuscript preparation and manuscript submission. XJW, ZHL, WTC and YZC as supervisors helped to perform the study analysis with constructive discussions, professional advice and revised the manuscript. All authors read and approved the final manuscript.

\section{Funding}

Project Supported by National Key Research and DevelopmentProgram of China (2016YFB0900100)

\section{Availability of data and materials}

Not applicable.

\section{Competing interests}

The authors declare that they have no competing interests.

\section{Author details}

'School of Electric Power, South China University of Technology, Guangzhou, China. ${ }^{2}$ Electric Power Research Institute of Guangdong Power Grid Co., Ltd, Guangzhou, China.

Received: 4 May 2019 Accepted: 30 May 2019

Published online: 19 June 2019

\section{References}

1. Zemin, J. (2008). Development of IT industry in China in the new age [J]. Shanghai Jiaotong Univ (Sci), 42(10), 1589-1607.

2. Zhitao, G., Jing, L., Longfei, W., et al. (2017). Achieving efficient and secure data Acquisition for Cloud-supported Internet of things in smart grid [J]. IEEE Internet Things J, 4(6), 1934-1944.

3. Al-Fuqaha, A., Guizani, M., Mohammadi, M., et al. (2015). Internet of things: A survey on enabling technologies, protocols and applications [J]. IEEE Communications Surveys \& Tutorials, 17(4), 2347-2376.

4. Reka, S. S. \& Dragicevic, T. (2018). Future effectual role of energy delivery: A comprehensive review of internet of things and smart grid [J]. Renew Sust Energ Rev, 91, 90-108.

5. Ahmed, E., Yaqoob, I., Hashem, I. A. T., et al. (2017). The role of big data analytics in internet of things [J]. Comput Netw, 129(2), 459-471.

6. Ou, H., Zeng, L., Li, X., et al. (2012). Power networking overview and development status [J]. Digital Communication, 39(5), 62-64.

7. Mengchun, J., Jiye, W., Zhihua, C., et al. (2014). Research and application of sensor information model in power internet of things [J]. Power System Technology, 38(2), 532-537.

8. Haoyong, C., Xiaojuan, W., Yongzhi, C., et al. (2018). Distributed sensing and cooperative estimation/detection of complex power/energy and energy systems []]. Power System Protection and Control, 46(18), 1-10.

9. Ping, Z., Shilin, L. I., \& Zheng, H. U. (2018). Enhanced-mobile ubiquitous smart environment-CThe super state of internet of things [J]. Chinese Journal on Internet of Things, 2(01), 17-23.

10. Weifu, Z., Jinghui, C., Xiaofeng, W., et al. (2014). The security analysis and countermeasure of power internet of things [J]. Electric Power Information and Communication Technology, 12(8), 121-125.

11. Yan, Z., Lingkang, Z., \& Xi, C. (2011). Architecture of power internet of things [J]. Electricity, 22(5), 10-15.

12. Murtaza, T., \& Andrea, C. (2011). Distributed and decentralized multicamera tracking [J]. Signal Processing Magazine, 28(3), 46-58.

13. Cai Yongzhi, A. (2016). Dissertation submitted for the degree of doctor of philosophy [D]. South China University of. Technology.

14. Leung, H., Chandana, S., \& WEI, S. (2008). Distributed sensing based on intelligent sensor networks [J]. IEEE Circuits \& Systems Magazine, 8(2), 38-52.

15. Li, K., Weixin, X., Jianjun, H., et al. (2013). Distributed compressive sensing for wireless sensor networks [J]. J Signal Process, 29(11), 1560-1567.

16. Faragher, R. (2012). Understanding the basis of the Kalman filter via a simple and intuitive derivation [lecture notes] [J]. Signal Processing Magazine, 29(5), 128-132.

17. Shanxiong, C., Zhongshi, H., Hailing, X., et al. (2015). A reconstruction algorithm of wireless sensor signal based on compressed sensing [J]. Chinese Journal of Computers, 38(03), 614-624.
18. Bing, S., \& Xinyu, J. (2014). Applications of compressive sensing in target tracking of wireless sensor networks [J]. Journal of Electronic Measurement \& Instrumentation, 28(05), 463-468.

19. Donoho, D. L. (2006). Compressed sensing [J]. Transactions on Information Theory, 52(4), 1289-1306.

20. Huazi, Z. (2013). Distributed signal processing and information spreading in wireless network [D]. Zhejiang University.

21. Heinzelman, W. R., Chandrakasan, A., \& Balakrishnan, H. (2002). Energyefficient communication protocol for wireless microsensor networks [C]. In Hawaii international conference on system sciences (p. 8020).

22. Falcão, D. M., Wu, F. F., \& Murphy, L. (1995). Parallel and distributed state estimation [J]. Transactions on Power Systems, 10(2), 724-730.

23. Predd, J. B., Kulkarni, S. R., \& Poor, H. V. (2007). Distributed learning in wireless sensor networks [M]. Chichester: John Wiley \& Sons.

24. Tai, X. (2013). New state estimation techniques for smart power networks with communication constraints | NOVA. The University of Newcastle's digital repository [J]. Eur Neurol, 36(3), 125-133.

\section{Submit your manuscript to a SpringerOpen ${ }^{\circ}$ journal and benefit from:}

- Convenient online submission

- Rigorous peer review

- Open access: articles freely available online

- High visibility within the field

- Retaining the copyright to your article

Submit your next manuscript at $\boldsymbol{\nabla}$ springeropen.com 